\title{
Game elements improve performance in a working memory training task
} Manuel Ninaus ${ }^{1^{*}+}$, Gonçalo Pereira ${ }^{2 *}$, René Stefitz ${ }^{1}$, Rui Prada ${ }^{2}$, Ana Paiva ${ }^{2}$, Christa Neuper ${ }^{1,3}$, Guilherme Wood ${ }^{1}$

\author{
${ }^{1}$ Department of Psychology, University of Graz, Graz, Austria \\ \{manuel.ninaus; guilherme.wood; christa.neuper\}@uni-graz.at \\ rene.stefitz@edu.uni-graz.at; \\ ${ }^{2}$ INESC-ID and Instituto Superior Técnico, Universidade de Lisboa \\ Avenida Professor Cavaco Silva, Porto Salvo, Portugal \\ goncalo.pereira@gaips.inesc-id.pt, \\ rui.prada@tecnico.ulisboa.pt, \\ ana.paiva@inesc-id.pt \\ ${ }^{3}$ Laboratory of Brain-Computer Interfaces, Institute for Knowledge Discovery, \\ Graz University of Technology, Austria \\ *contributed equally \\ +corresponding author
}

\begin{abstract}
The utilization of game elements in a non-game context is currently used in a vast range of different domains. However, research on game elements' effects in cognitive tasks is still sparse. Thus, in this study we implemented three game elements, namely, progress bar, level indicator, and a thematic setting, in a working memory training task. We evaluated the impact of game elements on user performance and perceived state of flow when compared to a conventional version of the task. Participants interacting with game elements showed higher scores in the working memory training task than participants from a control group who completed the working memory training task without the game elements. Moreover, game elements facilitated the individuals' performance closer to their maximum working memory capacity. Finally, the perceived flow did not differ between the two groups, which indicates that game elements can induce better performance without changing the perception of being "in the zone", that is without an increase in anxiety or boredom. This empirical study indicates that certain game elements can improve the performance and efficiency in a working memory task by increasing users' ability and willingness to train at their optimal performance level.
\end{abstract}

Keywords: working memory, game elements, gamification, flow experience, cognitive performance

\section{Introduction}

It is assumed that game elements, such as thematic setting, progress bars etc. influence the interaction with an application. The implementation of game elements in several domains of our daily life is rising immensely. However, there is a lack of empirical studies examining the effects of game elements on behaviour and performance of users. Many available cognitive training applications do use game "like" elements, but research on the assumed beneficial effects of using game elements for cognitive tasks or cognitive training is lacking. Commercially available cognitive training systems often cannot support their impressive beneficial claims sufficiently with empirical evidence (e.g. [13]). When such cognitive training applications are used for diagnostics, extended periods of time or 
in an even more crucial context of rehabilitation of neurological patients, empirical validation of its components is necessary.

Successful cognitive training requires significant effort from the users. Thus, motivation is a key concept in using cognitive trainings systems or cognitive tests appropriately. Using game elements, such as points, setting or progression bars in this non-game context, which is commonly known as gamification [4], may promote user performance, motivation and flow. However, the implementation of game elements or specifically external rewards could also have negative impact on the intrinsic motivation of users (see for example [5]). Recently, Mekler and colleagues [6] examined the effect of game elements in an image annotation task, which required participants to add tags to several paintings. They did not find a significant effect of game elements on intrinsic motivation between two groups in different conditions, one with game elements and another without them. However, Mekler and colleagues [6] could show that participants who received game elements within the image annotation task generated significantly more tags in less amount of time compared to a control group without any game elements.

The effect of game elements or gamification on cognitive performance of basic cognitive functions is sparsely researched. Prins and colleagues [7] reported improved performance and motivation in children with attention deficit hyperactivity disorder (ADHD) for a working memory training with game elements compared to a regular working memory training. The present study aims to further explore the influence of game elements on cognitive performance of users. Therefore, two versions of a working memory training task were developed. In one, various game elements (a progress bar, a level indicator, and a thematic setting) were implemented, while in the other, a conventional version of the same working memory training task was used. The implementation of game elements into the working memory training task was guided by concepts of flow theory [8] to keep users engaged.

The state of flow is considered optimal [8] because it is driven by a high intrinsic motivation towards the activity that leads to personal positive experiences such as immersion, enjoyment, fulfilment and skill. Even though a flow state can have diverse beneficial psychological impacts on people we were especially interested on its impact on learning and the performance in a working memory training task. Webster and colleagues [9] explored this important link and verified that flow experience can have a positive impact on learning. Therefore, the present study examined effects of adding game elements to a conventional working memory training task on performance and flow. It was expected that users provided with a gamified version of a working memory training task would perform better and perceive more flow than users with a conventional working memory training task without the specifically implemented game elements.

\section{Background}

\subsection{Working memory}

Working memory is a cognitive system that enables humans to manipulate and recall a limited amount of retained chunks of information for a brief period of time [10] [11]. Training working memory is very plausible for patients (e.g. stroke, ADHD, etc.) as well as for healthy adult persons (peak-performance training). Working memory is a brain system that allows the human to manipulate and recall a limited amount of retained chunks of information for a brief period of time [10]. Numerous studies demonstrated that working memory is of central importance for acquiring knowledge (e.g. [12]) and is involved in a variety of complex cognitive tasks and abilities (e.g. [13]). Alloway and Alloway [14] showed that working memory is even a better predictor for academic success than intelligence. Thus working memory is also a strong predictor for reading and mathematical skills [15].

Literature on working memory training shows that core training of working memory is especially promising. Core training studies typically involve tasks using sequential processing and frequent memory updating components integrated with a design targeting domain-general working memory mechanisms [16]. One very common and successful approach of core training paradigms is the complex span task. Basic simple span tasks require the participant to remember and recall a number of items which have been presented without interruption. Whereas in complex span tasks participants have to execute a distractor task after each presented item. For instance, participants have to remember a sequence of one-digit numbers. These numbers are presented sequentially and after each number the participants have to solve an easy equation (e.g. $6+8=15$, true or false?) while maintaining the presented number(s) in their temporary memory. Complex span tasks are a reliable 
measure of complex cognition [1] and therefore used in this study to develop two versions of a working memory training task.

\subsection{Flow}

Flow or a state of flow, was introduced by Csikszentmihalyi [8] and occurs when a person is completely absorbed by its current activity. Flow is associated with a number of factors: sense of control, action awareness merging, loss of self-consciousness, concentration on activity, distorted sense of time and autotelic experience (intrinsically rewarding) dimensions. However, flow is not easy to achieve since it actually only corresponds to a narrow band of experiences [17]. If a given activity is very challenging but a person has low skill at performing it, instead of flow he/she is lead to a state of anxiety. Conversely, if the person faces a very easy activity and he/she has high skill at performing it, again flow is missed and a state of boredom might be reached. Moreover, flow experience can also be used to evaluate the quality of gameplay [18].

Kiili [19] introduced an adapted version of the theory of flow for virtual environments, specifically to game based learning. According to the theory, there are several main "flow antecedents" [20] that should be considered to achieve a state of flow and consequently its learning effects:

- Clear Goals - clear goals facilitate the learner's focus on the activity which is related to a higher probability of experiencing flow;

- Feedback - helps the learner monitor his/her performance and progress and also avoid distractions, both related to a higher probability of experiencing flow;

- Playability - overly complex activities or interaction with the learning system are related with a lower probability of experiencing flow;

- Sense of Control - the learner's perception that he/she can develop his/her skills to reduce errors in challenges should be supported. If such a balance between the challenges and skills is offered there is a higher probability of experiencing flow.

Even though "Challenges" and "Skills" were not specifically described they are both fundamental antecedents. These antecedents are actually the key elements of the theory and are better characterized as a comparative dichotomy (e.g. Playability and Sense of Control antecedents).

\section{Game and Task Description}

\subsection{Cognitive Training Task}

The application (working memory task with game-elements GAME-version; working memory task without game elements NOGAME-version) was designed to guide the player through cycles of a complex span task activity for 25 minutes. The activity is composed by a sequence of phases that are typically (assuming an error free progression) presented in the following order (Figure 1):

- $\quad$ digit presentation $(D P)$ the application displays a one-digit number (key-digit) that the player must memorize until the unlocking phase;

- $\quad$ decision task $(T)$ the application presents a simple arithmetic decision task (e.g. $5+6=11)$ that the player must classify as either true or false (see Figure 2 GAME \& NOGAME, A);

- $\quad$ unlocking $(U)$ the player is asked to recall the key-digit initially presented;

This complex span task activity can be parameterized in terms of difficulty by varying the number of $D P \rightarrow T$ phase pairs before reaching the $U$ phase. The digits to recall in this phase are all those that were previously presented in each $D P$ phase. The number of digits to remember is therefore directly proportional to the number of $D P \rightarrow T$ pair sequences that precede it. 


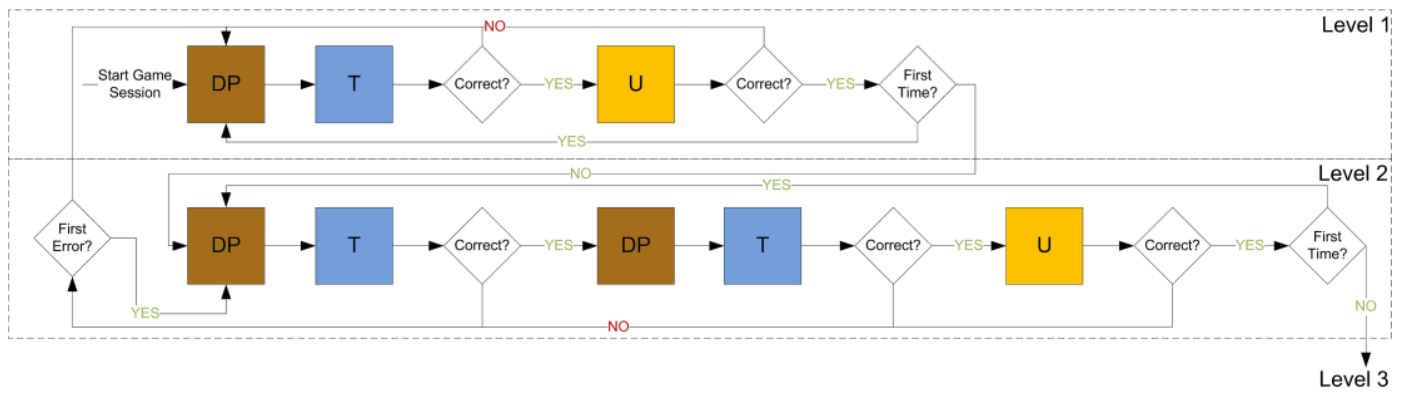

Figure 1. Components of flowchart for level 1 and 2. DP - Digit Presentation; T - Decision Task; $\mathrm{U}-$ Unlocking.

A user initiating a new session starts at level 1. Each level is composed by two sequential and equally difficult complex span task activities. If a player is able to successfully complete two equally difficult tasks then the user advances to the next level. For each level that the player advances one more $D P \rightarrow T$ pair, relatively to the previous level, is added to the complex span task. For example, in level 1 the player is presented with the following complex span tasks composed by the following phases: $D P \rightarrow T \rightarrow U$. However, in level 2 he/she faces tasks with the following phases: $D P \rightarrow T \rightarrow D P \rightarrow T \rightarrow U$. That is, for level $\mathrm{n}$ a player faces $\mathrm{n}$ phase pairs $D P \rightarrow T$ before entering phase $U$.

As in any cognitive task, the user can make mistakes and in the specific case of this complex span task they can occur either at the $T$ phase or at the $U$ phase. An error in any of these phases results in the player restarting the current complex span task level from the beginning. However, if the player makes two mistakes, in the same level, he/she levels down and starts a complex span task with a lower difficulty. This procedure allows the players to train at the optimal level of their capabilities. Furthermore, the adaptive difficulty of the task should ensure maximum performance of the users. Specifically, if a user is able to solve a specific level the user will advance into next level (increase in difficulty), but will level down (decrease difficulty) if the user is not able to correctly complete the task (recall the key-digit sequence). In the long run, the user will find his/her optimal performance range. This is the case for the working memory task with game elements (GAME) as well as the version without game elements (NOGAME). The flowchart presented in Figure 1 represents the possible paths in player progression according to the different phases of levels 1 and 2.

\subsection{GAME vs NOGAME}

The gamified version of the working memory training task (GAME) is presented to the player as a personal quest of freeing one's own brain areas (for a more detailed description see also [21]), while the version without game elements (NOGAME) is introduced as a regular working memory training task. The gamified version of the working memory training task provides a constantly visible progress bar at the top of the screen as well as a written indicator reflecting the current level of the user at the bottom of the screen (see Figure 2).

In the unlocking phase $U$ of the application users of the gamified version try to help freeing certain areas of the brain by remembering the correct numbers. That is, over the course of the 25 minutes of working memory training users in the GAME-group successively unlock different areas of the brain. This is visualized by a coloured brain region which is visually overlaid by a lock. When typing in the correct numbers the lock opens (see Figure 2 GAME, D) and the formerly locked brain area is freed and is shortly highlighted by intensifying its colour. In contrast users of the NOGAME group are only provided with a red " $\mathrm{X}$ " for wrong answer (see Figure 2 NOGAME, B) and a green check mark for a correct answer (see Figure 2 NOGAME, D).

In the gamified version of the working memory training task the user receives visual feedback of correct (growing neuron animation) or wrong (shrinking neuron animation) answer according to the result of the $T$-phase (see Figure 2 GAME, B), while in the non-game version only an " $\mathrm{X}$ " for wrong answer (see Figure 2 NOGAME, B) or a green check mark for a correct answer is shown (see Figure 2 NOGAME, D). The duration of the feedback and all other elements did not differ between the two versions of the application.

According to Kiilis [19] adapted version of the theory of flow for virtual environments and game based learning no differences between the GAME and NOGAME version can be expected at the level of "Challenges" and "Skills", since the working memory training task and its mechanics (including the adaptive difficulty system) did not differ between the two versions. Nevertheless, both versions of the working memory training task did provide these two fundamental antecedents of flow 
and therefore users should be able to get into a state of flow. However, the two versions of the working memory training task did differ in some of the other main "flow antecedents" [20] due to the implementation of specific game elements.

Both versions of the working memory task provided "clear goals". The main goal was of course to perform as good as possible in the working memory task and therefore the structure and the general mechanics of the working memory task were the same in both groups. However, as mentioned above, the overall setting of the working memory training differed in the two versions (see Figure 2 GAME vs. NOGAME). The working memory task with game elements had a more visually appealing design, provided an overall setting and setting specific feedback for correct and wrong answers. This difference could influence the perceived flow, overall impression and importance of the task.

Furthermore, the GAME version provided more precise "feedback" and a higher quantity of "feedback" (progress bar, level indicator; see Figure 2 GAME, A), which should influence the flow and performance of users [22] [23]. In the NOGAME version of the working memory training task users only had two feedback channels (green check mark for correct answer; red " $\mathrm{X}$ " for wrong answer).

In terms of "playability", clicking buttons and pressing keys on the keyboard, and "sense of control" ("Challenges" and "Skills") the working memory training task with and without game elements did not differ substantially. 


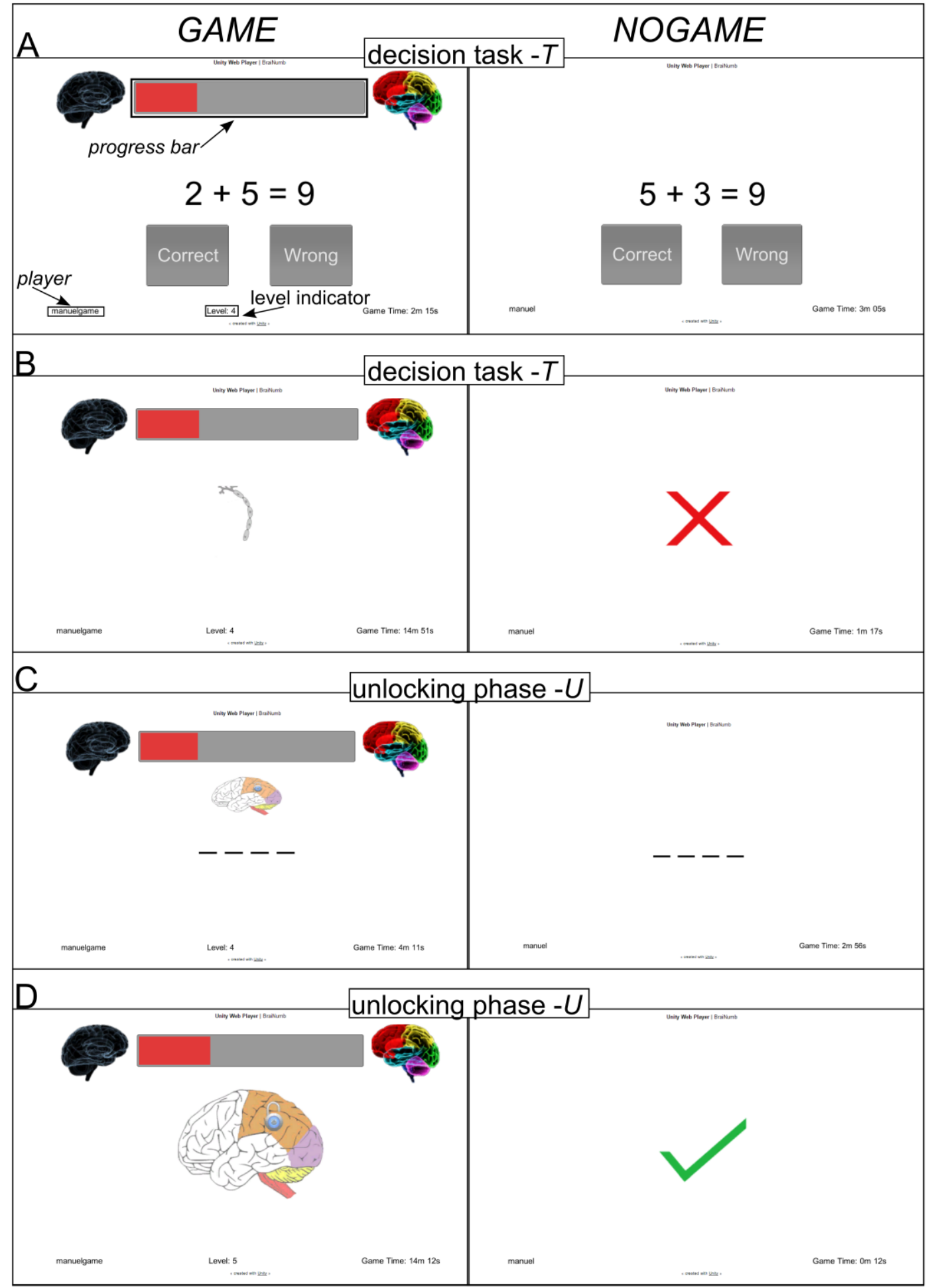

Figure 2. Examples of the user interface showing some of the differences between the two versions of the working memory training task; Left column: GAME version of the working memory training task; Right column: NOGAME version of the working memory training task; upper half shows the Decision Task - T and the corresponding feedback (row A \& B); lower half shows the Unlocking phase $-\mathrm{U}$ and the corresponding feedback (row $\mathrm{C} \& \mathrm{D}$ ) 


\subsection{Adaptive point score}

The used scoring system was designed to reward users adaptively throughout the whole application (1). A first layer of adaption is embedded into the level score formula (see $L S$ (level)) by using a Napierian logarithm of the current level. Thus, the system initially gives the user more score for each level increase. However, as the application progresses and becomes more difficult, the user needs to clear more levels to receive an additional amount of score similar to that of a previous level. MultiplierS was kept constant at the value 20 and was determined through extensive pre-testing. Notice that the MultiplierS is a constant setup at parameterization time that scales the values of the score according to the game design requirements and does not differ between GAME- and NOGAME-group. However, as already pointed out above, in the NOGAME-group there was no progress bar shown.

A second layer of adaptation is achieved by including a memory mechanism in the score formula (see PlayerS(level,pMaxS)). The current score then depends on two factors: the current level and the maximum score achieved by the user. If users decrease their current level then their level score is bound to be lower than the previous current score. To motivate players for having been able to reach higher levels (even if temporarily) the point scoring system rewards them by retaining a percentage of the difference between the maximum score they achieved and the current level's score. Ret was kept constant at the value 0.2 and was determined through extensive pre-testing. Notice that the magnitude of the reward is setup at parameterization time through the Ret constant according to the desired game experience and does not differ between GAME- and NOGAME-group. If the current level score is not lower than the maximum then that is the current player score.

Thus, the point score unifies the actual level of the players/users by considering the maximum level the user has reached so far. For example, person X achieved a score of 35 and person $\mathrm{Y}$ achieved a score of 25 . However, the maximum level of both in the working memory task was 6 . In this case a higher score indicates that the participants predominantly trained at their optimal performance level until the end of the session. While the lower score indicates that person Y predominantly trained below his or her optimal performance level or person Y showed a high level variability.

This shows that both participants could have the same working memory capacity, in the sense of maximum retainable information chunks for a short period of time (maximum level), but show differences in the ability to allocate working memory capacity over a longer period of time. Figure 3 shows the typical score progression of a user. One can observe the initial rise in the score until sequence number 20, while sequence corresponds to the sequential number of unlocking (U) phases. After sequence number $\sim 30$ it seems that the user found his maximum performance threshold and stays more or less at the same score.

$$
\begin{gathered}
L S(\text { level })=\text { Multiplier } S * \ln (\text { level }) \\
\text { PlayerS }(\text { level, } p M a x S)=\left\{\begin{array}{c}
L S(\text { level })+(\text { pMaxS }-L S(\text { level })) * \text { Ret if } L S(\text { level })<p M a x S \\
\text { otherwise }(\text { level })
\end{array}\right.
\end{gathered}
$$

level - the player's current level

MultiplierS - parameter to adjust the points scale

LS - level score

PlayerS - the current player score

pMaxS - the maximum score reached by the player

Ret - a retention percentage for when the player loses score 


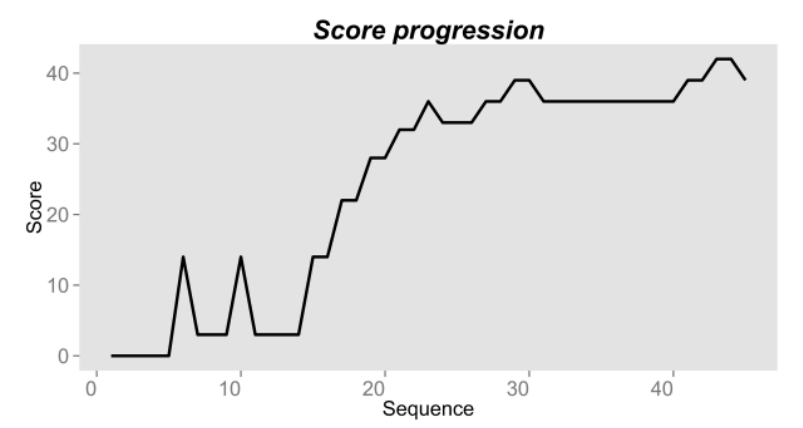

Figure 3. A typical example of score progression for one user; score = PlayerS (level,pMaxS) (adaptive point score); sequence $=$ sequence of unlocking $(\mathrm{U})$ phases, the individual maximum amount of sequences always corresponds to 25 minutes.

\section{Methods}

\subsection{Participants}

A total of thirty-nine university students were recruited for this study ( 7 male, 32 female; mean age $=23.8$ years, standard deviation $(S D)=5.05)$ and randomly assigned to one of two different versions of the online working memory test (with or without game elements). Eight participants were excluded from statistical analysis because of missing data. Thus, complete data sets for 13 participants in the group with game elements (GAME-group; 5 male, 8 female; mean age $=23.38$, $S D=3.80$ ) and 17 participants in the group without game elements (NOGAME-group; 1 male, 16 female; mean age $=24.12, S D=5.93$ ) were available. The participants were not aware of the aims of the study at the time of the data collection and gave their written informed consent. The study was approved by the ethics committee of the University of Graz and is in line with the Helsinki declaration.

\subsection{Material}

Flow experience was assessed with the Flow Short Scale (FKS "Flow-Kurzfragebogen", [24]). The FKS contains 10 items, which are related to 2 different subscales: "Absorption" and "automatic and self regulated process". The participants rate their experiences on a 7-point Likert scale from 1 (not at all) to 7 (completely). In this study we used the total value of all items, which provides a reliable measure of flow experience (Cronbach Alpha =0.90; [24]).

\subsection{Procedure}

Participants were recruited through flyers posted at the Campus at the University of Graz or via the university email-system. Participants responding to these were randomly assigned to either the online working memory task with implemented game elements (GAME-group) or the online working memory task without game elements (NOGAME-group). Participants received a written instruction about the procedure of the study and information about the working memory task. Afterwards, participants used a web-link to get to the corresponding online working memory task (GAME or NOGAME). After 25 minutes the online working memory task (GAME and NOGAME) ended and provided users of the GAME group feedback about their performance in form of achieved points (see Adaptive point score). Afterwards the online system provided a web-link to further online questionnaires assessing the flow experience of the users, and sociodemographic variables.

\subsection{Statistical Analysis}

R (http://www.r-project.org/) was utilized for all statistical analyses. For all analyses the alpha level was set to $\mathrm{p}=0.05$, two-tailed. To check for normal distribution of the dependent variables in the GAME and NOGAME group, Shapiro-Wilks tests were conducted. Bartlett's test was used to test for homogeneity of variances. To evaluate whether the performance in the working memory task, 
assessed using the point score of the application, and the flow experience (FKS) differed between GAME and NOGAME group, two two sample t-tests were calculated. Additionally, to compare the working memory capacity between the two groups the maximum level achieved in the working memory task was compared using a two sample t-test. Heatmaps were generated using the R plotting system ggplot2 (http://cran.r-project.org/web/packages/ggplot2/index.html).

\section{Results}

\subsection{Performance indicators of the working memory training task}

A two sample t-test comparing the point score of participants in the GAME group and the NOGAME group yielded a significant difference $(\mathrm{t}(28)=2.13 ; p<0.05$; Cohen's $d=0.79$; see Figure 4). Participants in the GAME group (mean $=34.46$; standard error $(S E)=0.93$ ) achieved higher scores than those in the NOGAME group (mean $=31.24 ; S E=1.11$ ). There was no significant difference between the GAME (mean $=7.00 ; S E=0.32$ ) and NOGAME group (mean $=6.35 ; S E=0.31)$ in the achieved maximum level $(\mathrm{t}(28)=1.44 ; \mathrm{ns})$.

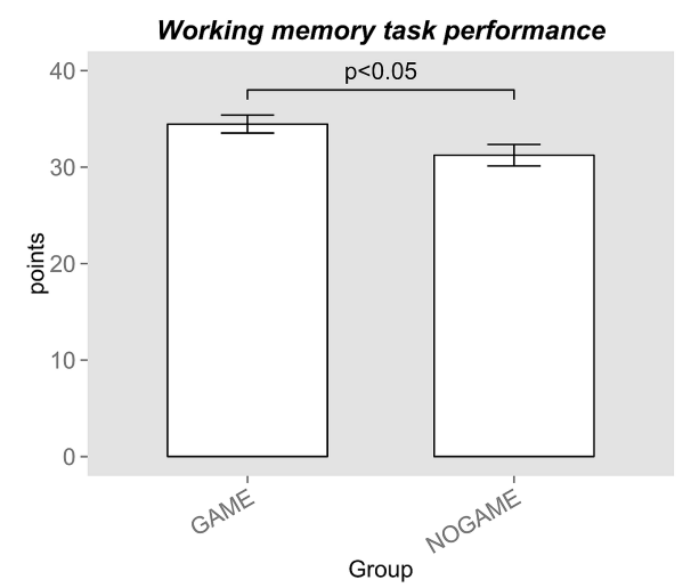

Figure 4. Task performance of working memory training task as assessed with the adaptive point score; bars represent the point score with standard error indicated for GAME and NOGAME group.

A heatmap of the level-progress of the GAME (left) and NOGAME (right) group is shown in Errore. L'origine riferimento non è stata trovata.. The colour of the heatmap indicates the number of participants in \%. The level of the users is plotted on $y$-axis while the sequence number on $\mathrm{x}$-axis corresponds to the sequential number of unlocking (U) phases. The performance of users can also be implicitly assessed by looking at the number of sequences (unlocking (U) phases). That is, a user who predominantly trained at a higher level would have worked on less number of sequences (unlocking (U) phases) than users who predominantly trained at a lower level. This can be explained by the fact that the absolute amount of time one user has for the cognitive working memory task is constant (25 minutes). However, the amount of sequences (unlocking (U) phases) is variable and depends on the average level of the user. That is, the time it takes to complete level 4 ( $4 \mathrm{x} D P \rightarrow T$ pairs and $1 U$-phase at the end) is about half the time it takes to complete level $8(8 \mathrm{x} D P \rightarrow T$ pairs and $1 U$-phase at the end), which means that users who train at a higher level complete less number of sequences (unlocking (U) phases) than those who train at a lower level. Thus, the provided heatmaps allow to visually examine the level progress of the users in the GAME and NOGAME group throughout the whole session of the working memory training task. The colour white reflects a low percentage of participants, while a high percentage of participants is reflected by the colour red. 


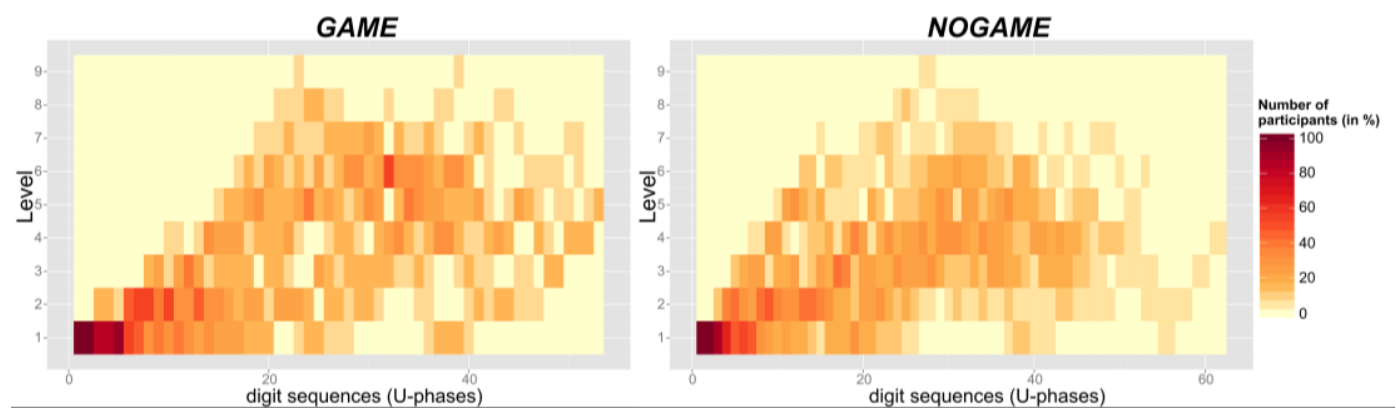

Figure 5. Heatmaps of GAME and NOGAME group; visualizes the level progress of the users in the GAME and NOGAME group throughout the whole session of the working memory training task; digit sequences $=$ sequence of unlocking $(\mathrm{U})$ phases, the individual maximum amount of sequences always corresponds to 25 minutes of training time; red = high number of participants, white $=$ low number of participants

\subsection{Flow}

To compare the perceived flow during the working memory task we used the scores of the FKS questionnaire of both groups. Participants' flow experience in the GAME group (mean $=44.54 ; S E$ $=2.82$ ) and the NOGAME group (mean $=40.06 ; S E=3.16$ ), as assessed with the FKS, did not significantly differ $(\mathrm{t}(28)=1.02 ; \mathrm{ns})$.

\section{Discussion}

The aim of the current study was to examine the effects of adding game elements to a conventional working memory training task on flow perception and performance. Thus, one group of participants interacted with a conventional working memory training task without game elements while the other used a working memory training task with added game elements. The results showed a significantly higher point score in the GAME group than in the NOGAME group, while there was no significant difference in the maximum achieved level between both groups. Additionally, the results revealed that there was no significant difference in the perceived flow between the GAME and NOGAME group.

\subsection{Performance}

The GAME group achieved a higher score in the application than participants in the NOGAME group. The point score was an adaptive measure, meaning that the score changed over the session of the working memory task, and took into account not only the maximum level but also the average capacity allocated over a longer period of time (for a detailed description see Game and Task Description). The adaptive point score also reflects how optimal or efficient the working memory task was carried out by the users throughout and until the end of the session. Users who worked at a much lower level than their maximum level were penalized with a lower point score.

The users of both groups did not significantly differ in their maximum achieved level for the working memory task, which indicates that the users did not differ in the number of maximum retainable information chunks. This result suggests that users of the GAME and NOGAME group did not differ in their maximal working memory capacity. Therefore, the higher point score in the GAME group compared to the NOGAME group cannot be explained by working memory capacity at all. Taking into account both results, maximum level and point score, it seems that the game elements enhanced the training performance and efficiency of the users.

The heatmap depicts the level progress of the users in the GAME and NOGAME group in the course of the working memory training session. By doing so, one can identify that users of the GAME group predominantly trained at a higher level compared to the NOGAME group. The visual inspection of the both heatmaps showed that users in the GAME group clustered at level 5-6, while users of the NOGAME group predominantly clustered at level 4-5. Furthermore, the heatmaps showed that in both groups the highest achieved level was level 9. Taken together, the heatmaps support the results of the inferential statistical analyses of point score and maximum level. Specifically, it can be reasoned that participants of the GAME group could better allocate working memory resources, 
through the active and real-time update and reset mechanisms [25] [26] which are more relevant components of working memory performance than mere maximal capacity.

The presented results for the performance indicators of the working memory task, maximum level, adaptive point score and heatmap, show that the implementation of specific game elements improve the users overall training performance. However, it is not clear from this study if the identified effect can be replicated in a long-term study. The current study presented results from a one-time interaction with the working memory training task without the intention to conduct a comprehensive training study with several training sessions for each user. Nevertheless, the study shows that the mere addition of game elements to a conventional working memory training task improves the efficiency and performance of the users.

Our results are in line with previous findings. Firstly, Mekler and colleagues [6] demonstrated the beneficial effects of game elements on the performance of an image annotation task. Secondly, Prins and colleagues [7] showed that ADHD children did benefit from a game version of a working memory training task compared to a non-game version. Further long term training studies and comprehensive cognitive assessments are needed to examine the beneficial effects of game elements on the performance of adult users in a working memory training task. Moreover, it is not clear, which of the various game elements contributed to the superior training efficiency of the GAME group. Therefore, systematic studies on the specific contributions of single game elements can also be of interest.

\subsection{Flow}

Several studies have shown that flow is associated with better performance (e.g. [27-31]). However, flow does not necessarily cause higher performance [27]. Specifically, in a working memory training task, that reinforces users to constantly interact with the task at their maximum capabilities and even beyond. Therefore, it is difficult to reach higher flow when someone is constantly between maximal performance and cognitive overload. Contrary to our expectations, the current study did not show a significant difference in the perceived flow between GAME and NOGAME group. On the one hand, this result supports our claim that game elements in a working memory task improve the performance and efficiency of the users. This rules out the explanation that the higher performance of the GAME group is caused by a difference in the perceived amount of flow between the two groups. On the other hand, we need to reconsider our expectations/hypotheses regarding the influence of game elements on the perceived flow in a working memory task. The equally high perception of flow in the two groups could also indicate that game elements can induce better performance without changing the perception of being "in the zone", that is, without increasing anxiety or boredom in the GAME group.

We did expect a higher flow in participants who used the working memory task with specifically implemented game elements compared to a group of participants who used the conventional version of the same task. Even though, the two versions (GAME and NOGAME) of the working memory task were identical regarding the basic structure of the task, they did differ in some of the flow antecedents [6].

Contrary to the conventional working memory training task (NOGAME) the gamified version of the working memory task provided a story/setting, namely the quest to free one's own brain. The process of "freeing one's own brain" was narratively supported by brain-themed animations (for details see Game and Task Description). We did assume that this thematic setting would influence the users' perception of the working memory training task. The users should perceive the gamified version as more intrinsically rewarding and autotelic and thus perceive a higher flow experience. It seems that this setting or story was not strong enough or not clearly enough communicated to the users to influence the overall impression and importance of the task. Specifically, it has been shown that the relationship between flow and challenge-skill balance is moderated by the perceived importance of the activity [27]. More research needs to be done to understand the influence of such contextual factors on flow.

A further distinguishing factor between the GAME and NOGAME version was the quantity and precision of the feedback elements. The GAME version provided a higher quantity of feedback and more precise feedback than the NOGAME version of the working memory task. In both versions of the task the application provided immediate feedback e.g. showing a locked brain area (GAME) or a red "X" (NOGAME) for a wrong answer. However, the gamified version provided a progress bar and a level indicator as additional feedback. Feedback is an important antecedent of flow and helps the user to monitor his/her performance [8][19][20]. It seems that, at a given basic level of feedback additional feedback modalities do not increase flow substantially. 
Generally, the current results suggest that game elements do not interfere with perceived flow in a very pronounced way. However, since the implemented game elements (progress bar, level indicator, and thematic setting) do not comprise the whole spectrum of possible game elements we cannot rule out the possibility that more or even different game elements could alter flow perception in a working memory task. Furthermore, even though flow theory provides several well defined antecedents each individual's experience of flow is unique as it also depends on personal characteristics such as emotions, values and previous experiences [20]. In this attempt to evaluate the effects of game elements on the performance in a working memory training task we did not use any personality-trait tests. Yet, the specific effects of such variables should be examined in future research. Furthermore, future studies need to account for individual differences in feedback and reward preferences, which can influence enjoyment and performance of the users [32].

\subsection{Conclusion}

This study provides empirical evidence that the implementation of certain game elements into a conventional working memory task can improve the performance and efficiency of users. There was no difference in flow between users who received the conventional working memory task and participants who used a gamified version of the same working memory task. The current results suggest that the mere addition of simple game elements can improve one's performance and willing to train on an optimal level. Users who received the conventional working memory task predominantly trained their working memory below their maximum capabilities, whereas participants using the gamified working memory task predominantly trained near their maximum capabilities. Nevertheless, future studies will be necessary to examine if the beneficial effects of game elements on the performance and efficiency in a working memory training will still be present in a long term training study. Furthermore, it is not clear which specific game elements influence the performance and perception of flow in a cognitive test and which cognitive abilities can even benefit from game elements. Overall, the results of this study can have wide-reaching practical implications in terms of the empirical evaluation and validation of game elements in the design of cognitive training and cognitive diagnostic. Gamification can be a cost-effective way to maximize performance in peak performance cognitive training.

\section{Acknowledgements}

This work is supported by GaLA (Games \& Learning Alliance) Network of Excellence funded by the EU in FP7-ICT-2009-5 under grant agreement no. 258169, by FCT(INESC-ID multi annual funding) under project PEst-OE/EEI/LA0021/2013 and FCT scholarship SFRH/BD/66663/2009.

\section{References}

[1] Shipstead Z., Hicks K. L., and Engle R. W., Cogmed working memory training: Does the evidence support the claims?, Journal of Applied Research in Memory and Cognition, vol. 1, no. 3, pp. 185-193, Sep. 2012. http://dx.doi.org/10.1016/j.jarmac.2012.06.003

[2] Hulme C. and Melby-Lervåg M., Current evidence does not support the claims made for CogMed working memory training, Journal of Applied Research in Memory and Cognition, vol. 1, no. 3, pp. 197-200, Sep. 2012. http://dx.doi.org/10.1016/j.jarmac.2012.06.006

[3] Owen A. M., Hampshire A., Grahn J. a, Stenton R., Dajani S., Burns A. S., Howard R. J., and Ballard C. G., Putting brain training to the test., Nature, vol. 465, no. 7299, pp. 775-8, Jun. 2010. http://dx.doi.org/10.1038/nature09042

[4] Deterding S., Dixon D., Khaled R., and Nacke L., From game design elements to gamefulness: defining gamification, in MindTrek 2011 Proceedings of the 15th International Academic MindTrek Conference: Envisioning Future Media Environments, 2011, pp. 9-15.

[5] Deci E., Koestner R., and Ryan R., A meta-analytic review of experiments examining the effects of extrinsic rewards on intrinsic motivation., Psychological bulletin, 1999.

[6] Mekler E. D., Brühlmann F., Opwis K., and Tuch A. N., Do Points , Levels and Leaderboards Harm Intrinsic Motivation? An Empirical Analysis of Common Gamification Elements, in Gamification 2013 Proceedings of the First International Conference on Gameful Design, Research, and Applications, 2013, pp. 66-73. 
[7] Prins P. J. M., Dovis S., Ponsioen A., ten Brink E., and van der Oord S., Does computerized working memory training with game elements enhance motivation and training efficacy in children with ADHD?, Cyberpsychology, behavior and social networking, vol. 14, no. 3, pp. 115-22, Mar. 2011.

[8] Csikszentmihalyi M., "Flow: The psychology of optimal experience." Harper \& Row, 1990.

[9] Webster J., Trevino L., and Ryan L., The dimensionality and correlates of flow in humancomputer interactions, Computers in human behavior, vol. 9, no. 4, pp. 411-426, 1994. http://dx.doi.org/10.1016/0747-5632(93)90032-N

[10]Baddeley A., Working memory: looking back and looking forward., Nature reviews. Neuroscience, vol. 4, no. 10, pp. 829-39, Oct. 2003. http://dx.doi.org/10.1038/nrn1201

[11] Baddeley A., Working memory, Science, vol. 255, no. 5044, pp. 556-559, 1992. http://dx.doi.org/10.1126/science.1736359

[12] Pickering S. J., "Working Memory and Education.” Elsevier Academic Press, 2006.

[13] Klingberg T., Training and plasticity of working memory., Trends in cognitive sciences, vol. 14, no. 7, pp. 317-24, Jul. 2010. http://dx.doi.org/10.1016/j.tics.2010.05.002

[14] Alloway T. P. and Alloway R. G., Investigating the predictive roles of working memory and IQ in academic attainment., Journal of experimental child psychology, vol. 106, no. 1, pp. 209, May 2010. http://dx.doi.org/10.1016/j.jecp.2009.11.003

[15] Gathercole S. E., Alloway T. P., Willis C., and Adams A.-M., Working memory in children with reading disabilities., Journal of experimental child psychology, vol. 93, no. 3, pp. 265-81, Mar. 2006. http://dx.doi.org/10.1016/j.jecp.2005.08.003

[16] Morrison A. B. and Chein J. M., Does working memory training work? The promise and challenges of enhancing cognition by training working memory., Psychonomic bulletin \& review, vol. 18, no. 1, pp. 46-60, Feb. 2011. http://dx.doi.org/10.3758/s13423-010-0034-0

[17] Csikszentmihalyi M., "Beyond boredom and anxiety." Jossey-Bass Publishers, 1975.

[18] Kiili K., Perttula A., Lindstedt A., Arnab S., and Suominen M., Flow Experience as a Quality Measure in Evaluating Physically Activating Collaborative Serious Games, International Journal of Serious Games, vol. 1, no. 3, pp. 35-49, 2014. http://dx.doi.org/10.17083/ijsg.v1i3.23

[19] Kiili K., Digital game-based learning: Towards an experiential gaming model, The Internet and Higher Education, vol. 8, no. 1, pp. 13-24, Jan. 2005. http://dx.doi.org/10.1016/j.iheduc.2004.12.001

[20] Kiili K., de Freitas S., Arnab S., and Lainema T., The Design Principles for Flow Experience in Educational Games, Procedia Computer Science, vol. 15, pp. 78-91, Jan. 2012. http://dx.doi.org/10.1016/j.procs.2012.10.060

[21] Pereira G., Ninaus M., Prada R., Wood G., Neuper C., and Paiva A., Free Your Brain A Working Memory Training Game, in Games and Learning Alliance conference, 2014, pp. 1-10.

[22] Hattie J. and Timperley H., The Power of Feedback, Review of Educational Research, vol. 77, no. 1, pp. 81-112, Mar. 2007. http://dx.doi.org/10.3102/003465430298487

[23] Kluger A. and DeNisi A., The effects of feedback interventions on performance: a historical review, a meta-analysis, and a preliminary feedback intervention theory., Psychological bulletin, vol. II, no. 2, pp. 254-284, 1996. http://dx.doi.org/10.1037/0033-2909.119.2.254

[24] Rheinberg F., Vollmeyer R., and Engeser S., Die Erfassung des Flow-Erlebens [measuring flowexperience], in Diagnostik von Motivation und Selbstkonzept, Göttingen: Hogrefe, 2003, pp. 261-279.

[25] Oberauer K., Süß H., Schulze R., Wilhelm O., and Wittmann W. W., Working memory capacity - facets of a cognitive ability construct, Personality and Individual Differences, vol. 29, pp. 1017-1045, 2000. http://dx.doi.org/10.1016/S0191-8869(99)00251-2

[26] Oberauer K., Su H., Wilhelm O., and Wittman W. W., The multiple faces of working memory : Storage , processing, supervision , and coordination, vol. 31, pp. 167-193, 2003.

[27] Engeser S. and Rheinberg F., Flow, performance and moderators of challenge-skill balance, Motivation and Emotion, vol. 32, no. 3, pp. 158-172, Sep. 2008. http://dx.doi.org/10.1007/s11031-008-9102-4

[28] Nakamura J. and Csikszentmihalyi M., The concept of flow, in Handbook of positive psychology, Snyder C. and Lopez S., Eds. Oxford: Oxford University Press, 2005, pp. 89-105.

[29] Jackson S., Thomas P., Marsh H., and Smethurst C., Relationships between flow, self-concept, psychological skills, and performance, Journal of applied sport psychology, vol. 13, pp. 129153, 2001. http://dx.doi.org/10.1080/104132001753149865

[30] Puca R. M. and Schmalt H.-D., Task enjoyment: A mediator between achievement motives and performance, Motivation and Emotion, vol. 23, no. 1, 1999. http://dx.doi.org/10.1023/A:1021327300925 
[31] Schüler J., Arousal of flow-experience in a learning setting and its effects on exam-performance and affect, Zeitschrift für Pädagogische Psychologie, vol. 21, pp. 217-227, 2007. http://dx.doi.org/10.1024/1010-0652.21.3.217

[32] Nagle A., Wolf P., Riener R., and Novak D., The Use of Player-centered Positive Reinforcement to Schedule In-game Rewards Increases Enjoyment and Performance in a Serious Game, International Journal of Serious Games, vol. 1, no. 4, pp. 35-47, 2014. http://dx.doi.org/10.17083/ijsg.v1i4.47 